\title{
Fast Adaptive Beamforming with Smart Antenna for Radio Frequency Repeater
}

\author{
Chaoqun Wang ${ }^{1, a}$, Guohua Wei ${ }^{1}$ and Teng Wang ${ }^{1}$ \\ ${ }^{1}$ Research Institute of Radar Technology, School of Information and Electronics, Beijing Institute of Technology, Beijing 100081, China
}

\begin{abstract}
We present a fast adaptive beamforming null algorithm with smart antenna for Radio Frequency Repeater (RFR). The smart antenna system is realized by a Direction Of Arrival (DOA) Estimator, whose output is used by an adaptive beamforming algorithm to shape a suitable radiation pattern of the equivalent antenna; so that the co-channel interference due to retransmitting antenna can be reduced. The proposed adaptive beamforming algorithm, which has been proved by formulaic analysis and simulation, has a lower computation complexity yet better performance.
\end{abstract}

\section{Introduction}

In a wireless communication system, radio frequency repeater (RFR) is widely used to extend the network coverage due to easy setting-up, less expensive and without increasing the number of base station [1]. Block diagram of the repeater is shown in Fig. 1. As it depicted, RFR plays a transit role of signal enhancement; its basic function is a RF signal power amplifier. But RFR have a problem of signal feedback where the output of the retransmitting antenna is fed back to the benefactor antenna due to the high gain of the repeater. The feedback interference is amplified in the repeater in company with desired signals. Feedback and amplification process is repeated again and again, the repeater cannot work with the accumulation of the interference signal, finally $[2,3]$.

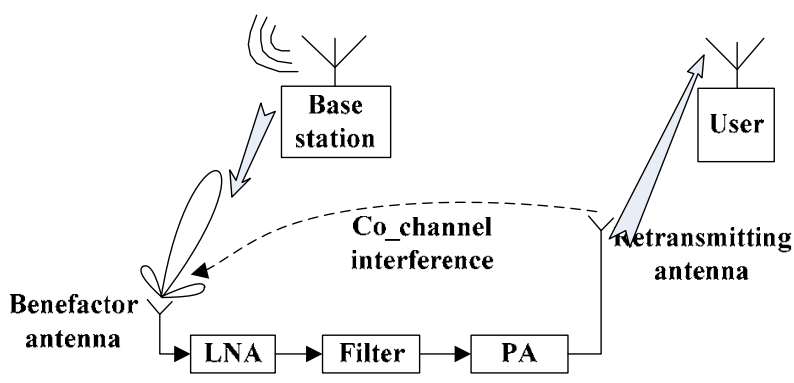

Figure 1. A radio frequency repeater with feedback interference signals.

There are many kinds of ways to solve this problem. The major method is to increase the isolation of benefactor antenna and retransmission antenna or reduce the gain of the repeater. But in fact, the former method usually increases the whole system cost greatly, and the latter can reduce the gain. So these two methods can't fundamentally solve the problem [4]. The second method is ICS (interference cancellation system) techniques. However, it requires lots of hardware complexity and computational delay [5-9]. To solve these problems, spatially adaptive filtering for RFR is studied, that is, with a certain shape of beam through the signal in the direction of the need to suppress the interference signal, which is also known as beamforming. In this paper, the beamforming algorithm based on minimum variance distortionless response (MVDR) criterion is studied. The traditional adaptive beamforming algorithm needs to construct covariance matrix of received signal and do matrix inversion directly, which is computationintensive and time-consuming [10-12]. Formulaic analysis and simulation demonstrate that the proposed adaptive beamforming algorithm has a lower computation complexity yet better performance.

\section{Adaptive BEAMFORMING}

\subsection{Smart antenna system}

The smart antenna system needs to differentiate the desired signal from the co_channel interferences and normally requires either the knowledge of a reference signal (or training signal), or the direction of the desired signal source. There exists a range of schemes to estimate the direction of sources with conflicting demands of accuracy and processing power. Similarly, there are many methods and algorithms to update the array weights, each with its speed of convergence and required processing time. The basic functional diagram of an adaptive beamformer is shown in Fig. 2.

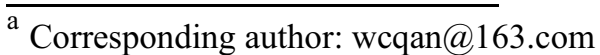




\subsection{Adaptive beamforming}

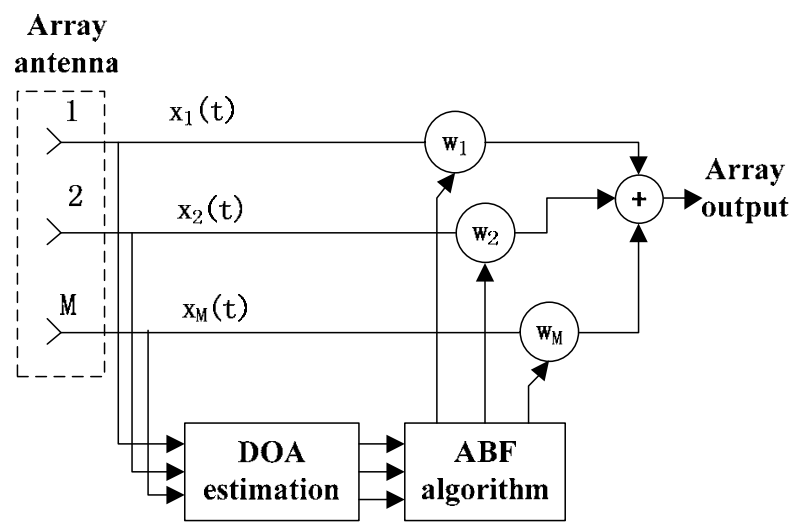

Figure 2. Functional block diagram of smart antenna system.

Adaptive beamforming is a commonly employed technique that enables system operation in an interference environment by adaptively modifying the system's antenna pattern so that nulls are generated in the angular locations of the interference sources. This approach is applicable to scenarios where multiple antenna elements are individually weighted to produce a desired directivity pattern. A typical method of forming the adaptive weights is via the MVDR algorithm, which implements a single linear constraint that maintains unit gain in the desired direction.

Considering a receiving antenna array with $\mathrm{M}$ array elements, the received signal is

$$
\boldsymbol{X}(t)=\boldsymbol{a}\left(\theta_{\mathrm{d}}, \varphi_{\mathrm{d}}\right) d(t)+\boldsymbol{a}\left(\theta_{\mathrm{e}}, \varphi_{\mathrm{e}}\right) e(t)+n(t)
$$

where $\boldsymbol{X}(t)=\left[x_{1}(t), x_{2}(t), \ldots, x_{\mathrm{M}}(t)\right]^{\mathrm{T}}$ is the complex vector of received signal; $d(t), e(t)$ and $n(t)$ are the desired signal, interference and noise components, respectively. Here $\boldsymbol{a}\left(\theta_{\mathrm{d}}, \varphi_{\mathrm{d}}\right)$ and $\boldsymbol{a}\left(\theta_{\mathrm{e}}, \varphi_{\mathrm{e}}\right)$ are the desired signal steering vector and the interference steering vector, respectively.

The traditional optimal weight vector of MVDR is

$$
W^{\mathrm{H}}=\boldsymbol{a}^{\mathrm{H}}\left(\theta_{\mathrm{d}}, \varphi_{\mathrm{d}}\right) R_{\mathrm{xx}}^{-1}\left[\boldsymbol{a}^{\mathrm{H}}\left(\theta_{\mathrm{d}}, \varphi_{\mathrm{d}}\right) R_{\mathrm{xx}}^{-1} \boldsymbol{a}\left(\theta_{\mathrm{d}}, \varphi_{\mathrm{d}}\right)\right]^{-1} .
$$

$R_{\mathrm{xx}}$ is the covariance matrix of received signal, whose size is $\mathrm{M} \times \mathrm{M}$. For large antenna array, the calculation quantity is considerable.

For RFR, the interference signal is a direct feedback from retransmission antenna to receiving antenna, so $e(t)$ can be expressed as

$$
e(t)=d(t) \times h(t)
$$

where, $h(t)$ is the system function of the interference system, which can be equivalent to an enlarged and timedelayed system. For the benefactor antenna array whose maximum size of the physical aperture is 1 meter, the far field requirement is more than 5 meters when the signal wavelength is $800 \mathrm{MHz}$. In the same repeater, the distance between the benefactor antenna and the retransmitting antenna can reach tens of meters, so the interferences can be regarded as plane waves.
The optimal weight vector of MVDR for RFR is the solution of the following optimization problem:

$$
\min W^{\mathrm{H}} Q W \text { subject to } W^{\mathrm{H}} \boldsymbol{a}\left(\theta_{\mathrm{d}}, \varphi_{\mathrm{d}}\right)=1
$$

where

$$
\begin{gathered}
N_{\mathrm{c}}(t)=\boldsymbol{a}\left(\theta_{\mathrm{e}}, \varphi_{\mathrm{e}}\right) e(t)+n(t) \\
Q=\mathrm{E}\left[N_{\mathrm{c}}(t) N_{\mathrm{c}}{ }^{\mathrm{H}}(t)\right] .
\end{gathered}
$$

Here $N c(t)$ is the combined noise (interference signal and system thermal noise) and $Q$ is the covariance matrix of the combined noise.

The optimization problem in (4) has an elegant closedformsolution given by:

$$
W^{\mathrm{H}}=\boldsymbol{a}^{\mathrm{H}}\left(\theta_{\mathrm{d}}, \varphi_{\mathrm{d}}\right) Q^{-1}\left[\boldsymbol{a}^{\mathrm{H}}\left(\theta_{\mathrm{d}}, \varphi_{\mathrm{d}}\right) Q^{-1} \boldsymbol{a}\left(\theta_{\mathrm{d}}, \varphi_{\mathrm{d}}\right)\right]^{-1} .
$$

The covariance matrix of the combined noise is

$$
Q=\boldsymbol{a}\left(\theta_{\mathrm{e}}, \varphi_{\mathrm{e}}\right) P_{\mathrm{e}} \boldsymbol{a}^{\mathrm{H}}\left(\theta_{\mathrm{e}}, \varphi_{\mathrm{e}}\right)+\sigma_{\mathrm{n}}^{2} I
$$

where $P_{\mathrm{e}}=\mathrm{E}\left[e(t) e^{\mathrm{H}}(t)\right]$ is the covariance matrix of interference, $\sigma_{\mathrm{n}}$ represents the power of noise, $I$ is an identity matrix.

According to the matrix inversion lemma, the inverse of covariance matrix can be written as

$$
\begin{gathered}
Q^{-1}=\left\{I-\boldsymbol{a}\left(\theta_{\mathrm{e}}, \boldsymbol{\varphi}_{\mathrm{e}}\right)\left[I+P_{\mathrm{e}} / \sigma_{\mathrm{n}}{ }^{2} \boldsymbol{a}^{\mathrm{H}}\left(\theta_{\mathrm{e}}, \varphi_{\mathrm{e}}\right) \boldsymbol{a}\left(\theta_{\mathrm{e}}, \varphi_{\mathrm{e}}\right)\right]^{-1}\right. \\
\left.\left.\times P_{\mathrm{e}} / \sigma_{\mathrm{n}}{ }^{2} \boldsymbol{a}^{\mathrm{H}}\left(\theta_{\mathrm{e}}, \varphi_{\mathrm{e}}\right)\right\} / \sigma_{\mathrm{n}}{ }^{2}\right\} .
\end{gathered}
$$

Substituting $Q^{-1}$ into (7), we can obtain the weight vector:

$$
W^{\mathrm{H}}=\Gamma \boldsymbol{a}^{\mathrm{H}}\left(\theta_{\mathrm{d}}, \varphi_{\mathrm{d}}\right)\left[I-\boldsymbol{a}\left(\theta_{\mathrm{e}}, \varphi_{\mathrm{e}}\right)\left(1 / I N R+r_{\mathrm{ee}}\right)^{-1} \boldsymbol{a}^{\mathrm{H}}\left(\theta_{\mathrm{e}}, \varphi_{\mathrm{e}}\right)\right] .
$$

where

$$
\begin{gathered}
\Gamma=\boldsymbol{a}^{\mathrm{H}}\left(\theta_{\mathrm{d}}, \varphi_{\mathrm{d}}\right)\left[I-\boldsymbol{a}\left(\theta_{\mathrm{e}}, \varphi_{\mathrm{e}}\right)\left(1 / I N R+r_{\mathrm{ee}}\right)^{-1} \boldsymbol{a}^{\mathrm{H}}\left(\theta_{\mathrm{e}}, \varphi_{\mathrm{e}}\right)\right] \\
\times \boldsymbol{a}\left(\theta_{\mathrm{d}}, \varphi_{\mathrm{d}}\right) \\
I N R=P_{\mathrm{e}} / \sigma_{\mathrm{n}}{ }^{2} \\
r_{\mathrm{ee}}=\boldsymbol{a}^{\mathrm{H}}\left(\theta_{\mathrm{e}}, \varphi_{\mathrm{e}}\right) \boldsymbol{a}\left(\theta_{\mathrm{e}}, \varphi_{\mathrm{e}}\right) .
\end{gathered}
$$

Here $\Gamma$ is the normalized coefficient, INR is the input interference to noise radio.

For large INR, (10) and (11) can be simplified as:

$$
\begin{gathered}
W^{\mathrm{H}}=\Gamma\left[\boldsymbol{a}^{\mathrm{H}}\left(\theta_{\mathrm{d}}, \varphi_{\mathrm{d}}\right)-r_{\mathrm{de}} r_{\mathrm{ee}}^{-1} \boldsymbol{a}^{\mathrm{H}}\left(\theta_{\mathrm{e}}, \varphi_{\mathrm{e}}\right)\right] . \\
\Gamma=\boldsymbol{a}^{\mathrm{H}}\left(\theta_{\mathrm{d}}, \varphi_{\mathrm{d}}\right)\left[I-\boldsymbol{a}\left(\theta_{\mathrm{e}}, \varphi_{\mathrm{e}}\right) r_{\mathrm{ee}}^{-1} \boldsymbol{a}^{\mathrm{H}}\left(\theta_{\mathrm{e}}, \varphi_{\mathrm{e}}\right)\right] \boldsymbol{a}\left(\theta_{\mathrm{d}}, \varphi_{\mathrm{d}}\right)
\end{gathered}
$$

where $r_{\mathrm{de}}=\boldsymbol{a}^{\mathrm{H}}\left(\theta_{\mathrm{d}}, \varphi_{\mathrm{d}}\right) \boldsymbol{a}\left(\theta_{\mathrm{e}}, \varphi_{\mathrm{e}}\right)$ is the spatial correlation coefficient between desired signal and interference. As $\boldsymbol{a}\left(\theta_{\mathrm{e}}, \varphi_{\mathrm{e}}\right)$ is a $\mathrm{M} \times \mathrm{J}(\mathrm{J}$ is the number of retransmitting antenna) matrix, $r_{\text {ee }}$ is a $\mathrm{J} \times \mathrm{J}$ matrix.

Two reasons are given to explain why the calculation of the proposed adaptive beamforming algorithm is faster than the traditional:

1) The matrix size of $Q$ and $r_{\mathrm{ee}}$ is $\mathrm{M} \times \mathrm{M}$ and $\mathrm{J} \times \mathrm{J}$, 
respectively. For large receiving antenna array, the latter is significantly smaller than the former when performing matrix inversion.

2) Since the position of retransmitted antenna relatived to benefactor antenna is known for RFR, the arrival of the interference signal is known. Therefore, $r^{-1}$ ee can be calculated and stored in advance.

\section{Simulation Results and Analysis}

In this section, a series of numerical examples will be presented to validate the efficiency of the proposed method. In a RFR system, there are a relatively large number of interferers compared to antenna elements. Normally, to simplify the analysis, only the strongest interferers are considered. For our simulation, we consider the case of one or two interferers only. We employ uniformly circular array (UCA) with eight antenna elements having a spacing of $\lambda$, where $\lambda$ denotes the signal wavelength. The geometry of UCA is shown in Fig. 3. Simulations are done for values of interference azimuths $\left(-30^{\circ}, 50^{\circ}\right)$ and interference pitch angle $\left(-35^{\circ}\right.$, $\left.10^{\circ}\right)$. The azimuth and pitch angle of desired signal are $10^{\circ}$ and $0^{\circ}$, respectively. The power of the input PN sequence is $-29.12 \mathrm{~dB}$, and the interference signal is $13.78 \mathrm{~dB}$ with the thermal noise is about $-70 \mathrm{~dB}$, that is, the input signal-to-interference-plus-noise ratio (SINR) is $-39.48 \mathrm{~dB}$.

The desired PN sequence is shown in Fig. 4 and parts signals received by the antenna array are showed in Fig. 5. This figure shows that the desired sequence is completely submerged by the interference signal, and then the receiving system cannot distinguish the useful signal. Under this circumstance, adaptive beamforming is necessary. Fig. 6 shows the comparison of error values of the output signal and the desired sequence between proposed algorithm and conventional MVDR algorithm. As we can see, these two methods can filter out the interference and recovery the desired signal, but the error values using the proposed algorithm is smaller than the conventional algorithm. The average error of the proposed algorithm is $-44.47 \mathrm{~dB}$, which is about $9 \mathrm{~dB}$ less than traditional algorithm.

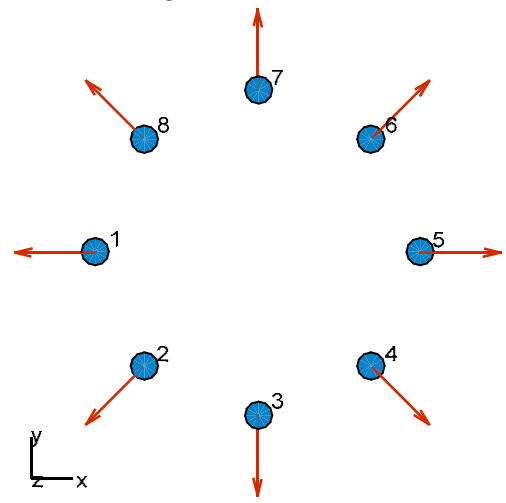

Figure 3. Geometry of UCA with eight antenna elements.

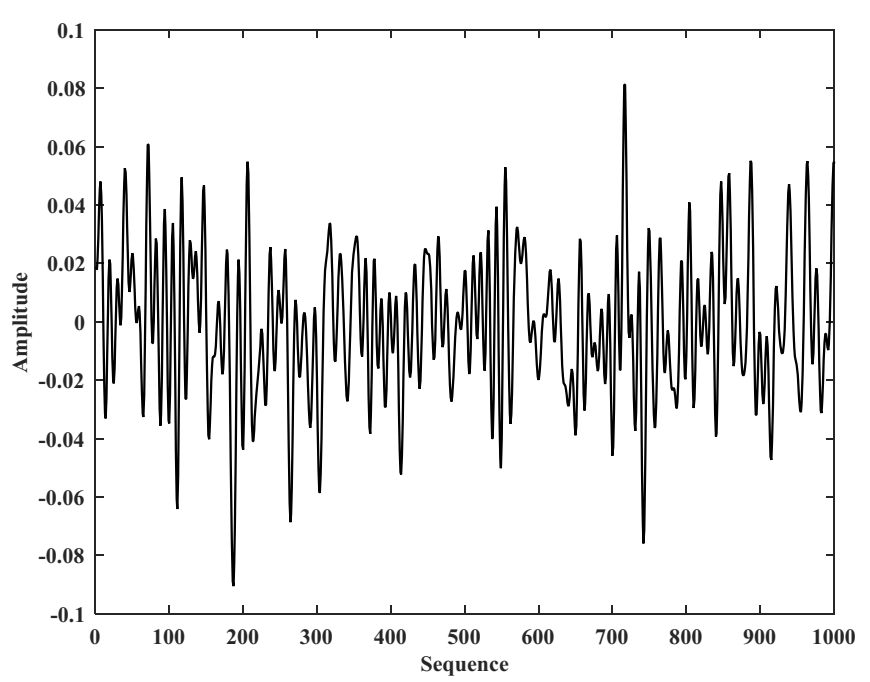

Figure 4. Desired PN sequence.
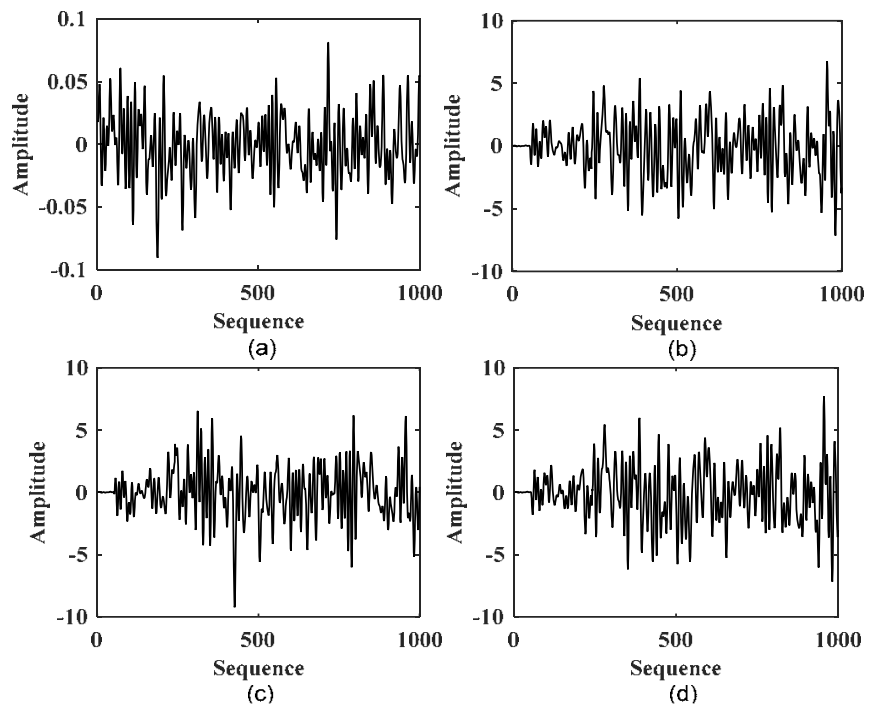

Figure 5. Comparisons for: (a) desired PN sequence, (b) signal received by the first antenna, (c) signal received by the second antenna and (d) signal received by the third antenna.

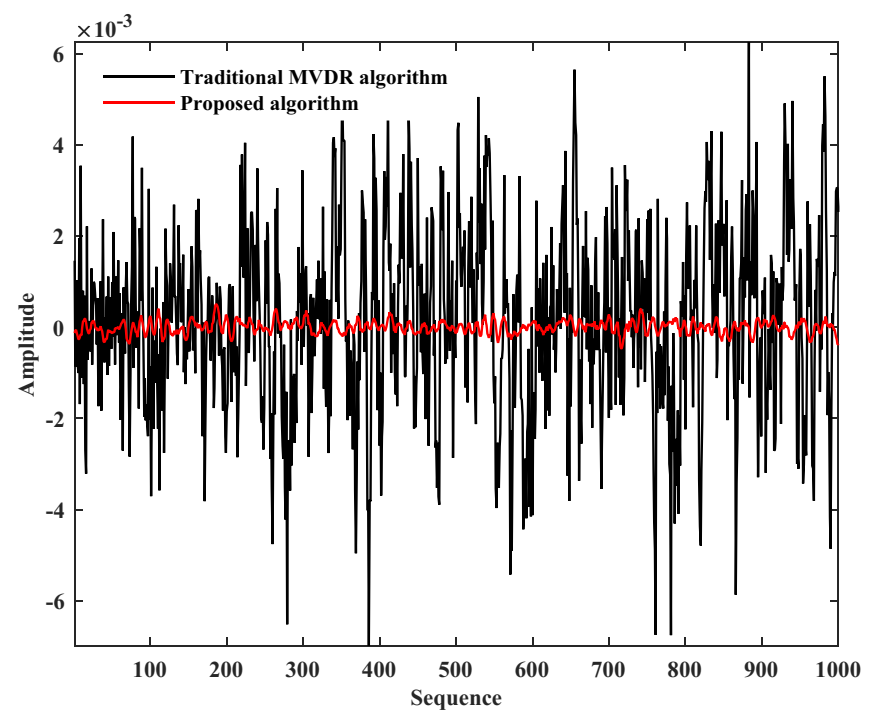

Figure 6. Comparisons of error values for: (a) traditional MVDR algorithm and (b) proposed algorithm. 


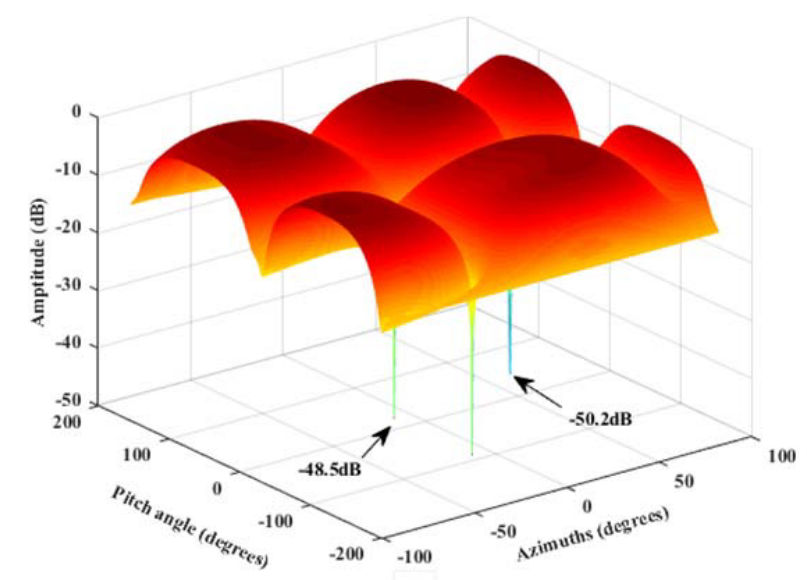

(a)

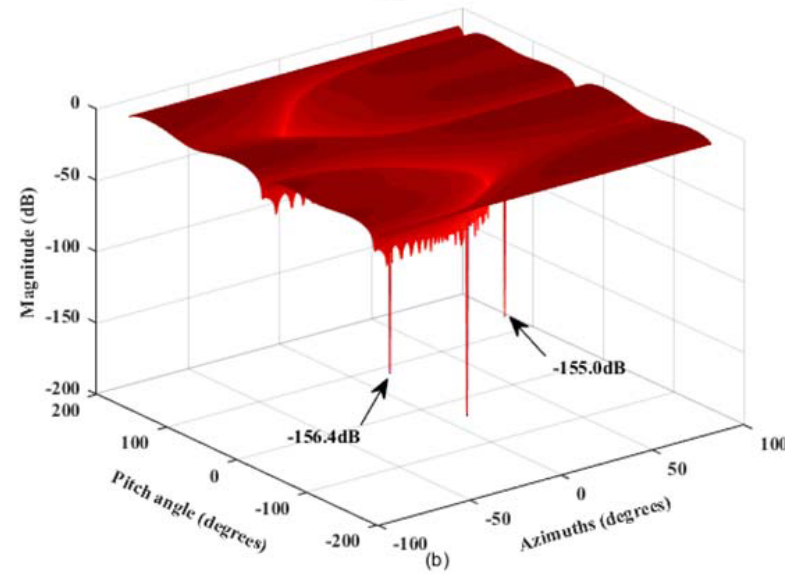

Figure 7. Antenna pattern formed by: (a) traditional MVDR algorithm, (b) proposed algorithm.

Fig. 7 compares the antenna pattern formed by traditional MVDR algorithm and proposed algorithm. There are two deeper nulls achieving -156.4 dB and $155.0 \mathrm{~dB}$ in the interference direction, as shown in Fig. 7(b). However, the nulls in Fig. 7(a) merely achieve $48.5 \mathrm{~dB}$ and $-50.2 \mathrm{~dB}$. The output SINR of the proposed algorithm is $46.28 \mathrm{~dB}$ that is $10 \mathrm{~dB}$ more than traditional MVDR algorithm. In summary, the proposed method has better performance.

In order to further validate the efficiency of the proposed method, different direction of arrival for desired signal is studied as shown in Fig. 8. As we can see, the main beam of antenna pattern is moving with the direction of arrival. At the same time, two deep nulls is still maintaining in the direction of interference $\left(70^{\circ}\right.$ and $\left.110^{\circ}\right)$.

\section{Conclusion}

In this paper, a fast adaptive beamforming algorithm based on MVDR criterion is studied for RFR. The analytic expression of the weight demonstrates the proposed adaptive beamforming algorithm is faster than the traditional, so it is more suitable for real-time application. Simulation results show that, in the ideal case, the nulls of the beam which are given by (15) can be completely zero, meaning that the interference direction can be fully zeroed. Meanwhile, this proposed algorithm can obtain better performance, compared with the traditional MVDR algorithm.

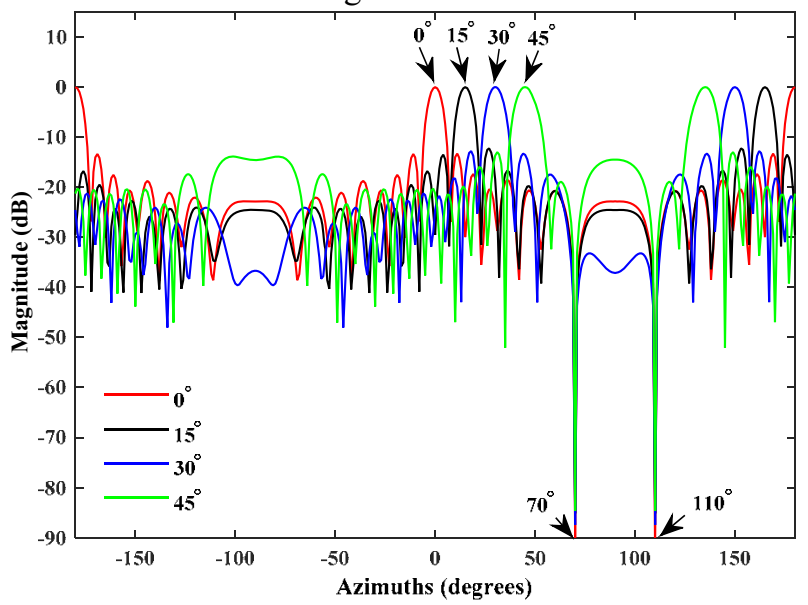

Figure 8. Antenna pattern at different direction of arrival for desired signal.

\section{References}

1. K. Salehian, M. Guillet, B. Caron, and A. Kennedy, "On-channel repeater for digital television broadcasting service," IEEE Trans. Broadcast., vol. 48, no. 2, pp. 97-102, Jun. 2002.

2. S. J. Kim et al., "Adaptive feedback interference cancellation system (AF-ICS)," in IEEE MTT-S Dig., Jun. 2003, pp. 627-630.

3. E. Ahmed and A. M. Eltawil, "All-digital selfinterference cancellation technique for full-duplex systems," IEEE Trans. Wireless Commun., vol. 14, no. 7, pp. 3519-3532, July. 2015.

4. J. Wei, L. Ren, and J. Chen, "Study on repeater with adaptive interference cancellation," in Proc. 8th WiCOM. Net. Mobile Computing, 2012, pp. 1-4.

5. R. N. Braithwaite and S. Carichner, "Adaptive echo cancellation for an on-frequency RF repeater using a weighted power spectrum," in Proc. 10th European Conf. Wireless Technol., Oct. 2007, pp. 82-85.

6. M. Lee, B. Keum, and Y. Serk Shim, "A scheme to compensate for the input multipath channel effect in an interference cancellation repeater for mobile communication systems," in Proceedings ATC, 2008, pp. 92-96.

7. T. Riihonen, S. Werner and R. Wichman, "Optimized gain control for single-frequency relaying with loop interference," IEEE Trans. Wireless Commun., vol. 8, no. 6, pp. 2801-2806, Jun. 2009.

8. E. Habets, J. Benesty, I. Cohen, S. Gannot, and J. Dmochowski, "New insights into the MVDR beamformer in room acoustics," IEEE Trans. Audio, Speech, Lang. Process., vol. 18, no. 1, pp. 158-170, Jan. 2010.

9. J. Y. Choi et al., "Interference cancellation techniques for digital on-channel repeaters in $\mathrm{T}$ DMB system," IEEE Trans. Broadcast., vol. 57, no. 1, pp. 46-56, Mar. 2011.

10. S. I. Park, Y. J. Lee, H. M. Kim, and H.N. Kim, "Equalisation digital on-channel repeater with a 
feedback interference canceller for the advanced television systems committee terrestrial digital television system," IEEE Trans. Commu., vol. 7, no. 16, pp. 1769-1776, Aug. 2013.

11. C. Pan, J. D. Chen, and J. Benesty, "Performance study of the MVDR beamformer as a function of the source incidence angle," IEEE/ACM Trans. Audio, Speech, Lang. Process., vol. 22, no. 1, pp. 67-79, Jan. 2014.

12. R. R. Qian, M. Sellathurai, and D. Wilcox, "A study on MVDR beamforming applied to an ESPAR antenna," IEEE Signal Process. Lett., vol. 22, no. 1, pp. 1070-9908, Jan.2015.

13. J. J. Blanz, A. Papathanassiou, M. Haardt, I. Furio, and P.W. Baier, "Smart antennas for combined DOA and joint channel estimation in time-slotted CDMA mobile radio systems with joint detection," IEEE Trans. VT., vol. 49, no. 2, pp. 293- 306, Mar. 2000.

14. S. Hwang, S. Burintramart, T. K. Sarkar, and S.R. Best, "Direction of arrival (DOA) estimation using electrically small tuned dipole antennas," IEEE Trans. Antennas Propagat., vol. 54, no. 11, pp. 3292-3301, Nov. 2006.

15. J. P. González and B. Rodríguez, "Interference rejection degradation in function of the DOA in a beamforming system," IEEE Latin America Trans., vol. 13, no. 1, pp. 48-53, Jan. 2005. 